\title{
Cognitive Behavioral Therapy for Insomnia: Is it Effective in Treating Symptoms of Comorbid Psychiatric and Medical Disorders? A Review
}

\author{
Sooyeon Suh, PhD, CBSM ${ }^{1,2}$ \\ 'Department of Psychology, Sungshin Women's University, Seoul, Korea \\ 2Department of Psychiatry and Behavioral Sciences, Stanford University, Palo Alto, CA, USA
}

Insomnia is a highly comorbid disorder with other psychiatric and physical illnesses. Cognitive behavioral therapy for insomnia (CBTI) is effective for treating insomnia symptoms. Additionally, it is also effective in treating symptoms of the comorbid psychiatric and physical illnesses, and is recommended as an adjunctive treatment to enhance treatment effects of the original disorder. This current article is a review of CBTI with comorbid physical and psychiatric disorders.

Sleep Med Res 2015;6(1):10-15

Key Words Insomnia, Comorbid psychiatric, Medical disorder.

Received: April 21, 2015

Revised: May 21, 2015

Accepted: May 21, 2015

\section{Correspondence}

Sooyeon Suh, PhD, CBSM

Department of Psychology,

Sungshin Women's University,

2 Bomun-ro 34da-gil,

Seongbuk-gu, Seoul 136-742, Korea

Tel +82-2-920-7215

Fax +82-2-920-2040

E-mail alysuh@sungshin.ac.kr

\section{INTRODUCTION}

Insomnia is defined as having 2 or more weeks of sleep difficulties, and approximately 9-18\% of American adults suffer from the disorder. ${ }^{1-6}$ The morbidity rate in Korea is similar to that in America, ranging from $10-17 \%{ }^{7}$ Chronic insomnia is associated with the following symptoms: daytime fatigue, decrease in memory and concentration, negative mood, lower quality of life, higher rates of absence from work, increase in medical costs, depression, and long-term consumption of sleeping pills. ${ }^{8-13}$

In the past, DSM-IV-TR categorized insomnia into two subtypes; primary insomnia and secondary insomnia. Primary insomnia was considered the most classical form of insomnia, which is learned or conditioned when sleep disturbance is caused by sleep-interfering behaviors and cognitive and physiological hyperarousal, including factors such as psychological distress and excessive worry about sleep. On the other hand, secondary insomnia is comorbid with other sleep, physical, or mental disorders. According to research by Ford and Kamerow, ${ }^{1}$ the largest number of people who have comorbid insomnia are those with psychiatric disorders. Based on research that most individuals with insomnia have comorbid disorders, DSM5, which was released in 2013, combined the two terms (primary and secondary insomnia) into "insomnia disorder."14 Based on the new DSM-5, clarification of the presence of comorbid disorders is mandatory.

\section{COGNITIVE-BEHAVIORAL THERAPY FOR INSOMNIA}

Cognitive-behavioral therapy for insomnia (CBTI) is the most effective non-pharmacological treatment for insomnia. Both the National Institute of Health Consensus Statement and British Association of Psychopharmacology recommends CBTI as the first line of treatment for insomnia. ${ }^{14,15}$ 
Cognitive-behavioral therapy for insomnia consists of five treatment components: sleep education, stimulus control, sleep restriction, relaxation techniques, and cognitive therapy. Therapists primarily utilize both cognitive and behavioral treatment elements to treat insomnia patients. See Perlis \& Lichstein, and Morin and Espie ${ }^{16}$ for in-depth details about CBTI.

Currently, the most common treatment for insomnia is using benzodiazepine receptor blockers and sedative antidepressants. However, CBTI is also widely acknowledged as an effective treatment for insomnia, and research has accumulated over the past 20 years about its effectiveness. In a meta-analysis by Smith et al. ${ }^{17}$ comparing pharmacological treatments to CBTI, results indicated that CBTI also had short-term effects that were similar to pharmacological treatment. Additionally, compared to pharmacological treatment, research indicates that CBTI maintained long-term treatment effects, and were also preferred more by insomnia patients. ${ }^{18-21}$ In another study that administered CBTI to chronic insomnia patients who were taking sleep medication, $23.5 \%$ of the patients successfully stopped taking their medication without specific instructions to decrease their medication intake. Patients also had a significant decrease in sleep onset latency, wake after sleep onset, severity of insomnia symptoms, and dysfunctional beliefs about sleep, in addition to an increase in sleep efficiency. To summarize, we can carefully conclude that CBTI can be an effective and adjunctive treatment option for individuals who are dependent on sleep medication. ${ }^{22}$

With the increasing number of studies showing the effectiveness of CBTI, there have been an increase in studies interested in showing how treating insomnia through CBTI can also alleviate symptoms from comorbid psychiatric disorders by treating insomnia. As mentioned above, a large proportion of insomnia patients also have comorbid psychiatric disorders. Based on criteria of the DSM-5, it is evident that many psychiatric disorders include sleep symptoms as a part of the diagnosis. For example, major depressive disorders lists "insomnia and hypersomnia every day" and "fatigue or loss of energy every day", and Substance Abuse and Mental Health Services (SAMSHA) lists insomnia as one of the 10 ten warning signs of individuals who are at high risk for suicide. ${ }^{23}$ In addition, "decreased need for sleep" is listed for bipolar disorder, while "sleep disturbance" and "being easily fatigued" is listed for generalized anxiety disorder. "Recurrent distressing dreams of the event" and "difficulty falling or staying asleep" are also listed as diagnostic criteria for post-traumatic stress disorder. Considering that many psychiatric disorders list sleep symptoms as part of diagnostic criteria, it is conceivable that treatment insomnia in individuals with psychiatric disorders may help alleviate symptoms of the comorbid disorder.

Similar research can be found with physical disorders. The prevalence of insomnia comorbid with chronic illnesses such as chronic pain, cardiovascular disease, diabetes, AIDS, and cancer ranges from $16-82 \%$, which is higher than the general pop- ulation. ${ }^{17,24-26}$ Based on previous research indicating sleep deprivation negatively affects the immune system, pain perception, metabolism, cognitive function and mood, CBTI is helpful for insomnia patients that have comorbid physical and mental illnesses for the following reasons. ${ }^{17}$ First, CBTI treats insomnia symptoms, which subsequently may lead to the alleviation of symptoms of comorbid physical and psychiatric illnesses. Additionally, treatment of insomnia may also prevent the recurrence of symptoms of comorbid illnesses, such as depressive episodes. Second, CBTI as a non-pharmacological treatment may help decrease the concerns of both patients and doctors regarding long-term use of sleep medication. Third, insomnia patients with comorbid disorders such as a history of substance abuse or chronic illness who take other medication for their original disorder may be able to avoid the risks of side effects and drug interactions from sleep medication.

Similarly, studies on comorbid insomnia has been receiving great attention, and studies investigating CBTI to alleviate symptoms of comorbid physical or psychiatric illnesses have been increasing. This paper will review currently published studies divided by psychiatric and physical illnesses, with the goal of helping guide effective treatment for clinicians.

\section{THE EFFECTIVENESS OF USING CBTI FOR COMORBID PSYCHIATRIC ILLNESSES}

\section{Depression}

More than half of depressed patients have difficulty falling asleep, wake up frequently during the night, or experience early morning awakenings. ${ }^{27}$ Insomnia symptoms, especially ones that appear early, are associated with depression symptom severity ${ }^{28}$ When insomnia is comorbid with psychiatric illnesses such as depression, treatment and management of the psychiatric illness becomes complicated. Because insomnia influences depression symptoms such as daytime depressed mood, negative mood, irritability, decreased motivation, and lack of concentration and concentration, depressed patients who have comorbid insomnia symptoms have worse symptomatology compared to those who do not have insomnia symptoms. ${ }^{29} \mathrm{In}$ somnia symptoms are the most common residual symptoms in depressed patients after receiving treatment, and up to $44 \%$ of depressed patients who completed treatment for depression report residual symptoms of insomnia. ${ }^{27,30}$ Additionally, insomnia treatment helps facilitate the effects of anti-depressants, and depressed patients who have comorbid insomnia symptoms show decreased treatment effects of anti-depressants.

Insomnia symptoms is a big risk factor for depression relapse in depressed patients. A study by Reynolds et al. ${ }^{31}$ found that the majority of depressed patients who had residual insomnia symptoms after receiving nortriptyline and interpersonal therapy re- 
lapsed a year after discontinuing pharmacotherapy. In contrast, $90 \%$ of depressed patients who did not have residual insomnia symptoms did not have depression relapse after one year. Depressed patients who have comorbid insomnia and receive treatment generally relapse within 4 months, while depressed patients who did not have insomnia patients relapsed after 12 months. ${ }^{32}$ Thus, depressed patients who have insomnia show faster rates of relapse compared to patients without insomnia.

Cognitive-behavioral therapy for insomnia can be helpful for depressed patients with insomnia, and depression symptoms can improve even in the absence of depression-specific treatment. A study by Manber et al. ${ }^{33}$ treated 30 depressed patients with comorbid insomnia with the anti-depressant escitalopram, and then randomly assigned half of those patients to receive group CBTI, while the other half did not receive CBTI. After 12 weeks of tracking depression symptoms, depressed patients who received a combination of an anti-depressant and CBTI had a significantly larger remission rates of depression (61.5\% vs. $33.3 \%$ ), and also larger decreases in insomnia symptoms (50\% vs. $7.7 \%$ ) compared to controls. Other studies have reported that insomnia patients who receive CBTI show a significant decreased on scores using the Beck Depression Inventory. ${ }^{34,35}$ This indicates that depression symptoms can improve using only insomnia treatment.

Another area that has received wide attention is the relationship between insomnia and suicide. There is ample evidence that insomnia increases the risk of suicidal behavior, suicidal ideation, suicidal attempts, and completed suicides. ${ }^{30,36,37}$ Based on these studies, SAMSHA lists insomnia symptoms as one of the 10 warning signs preceding suicide. Recently, Manber et al. ${ }^{38}$ and colleagues reported a study with 311 insomnia patients receiving 7 sessions of CBTI. Among these patients, 65 (23\%) reported "1 or higher" on the suicidal ideation item of the Beck Depression Inventory. However, a majority of these patients reported decreased suicidal ideation following CBTI treatment, and only $10 \%$ of the patients endorsed suicidal ideation after receiving treatment.

\section{Bipolar Disorder}

The sleep behavior of patients with bipolar disorder going through a major depressive episode is similar to sleep behavior patterns of patients with major depressive disorder. However, when these patients are in the midst of a manic episode, their total sleep time, slow wave sleep, and REM sleep decrease significantly, and sleep onset latency and REM density also increase. ${ }^{39,40}$ Additionally, medication that is typically used for bipolar disorder patients such as lithium and lamotrigine can cause insomnia symptoms. ${ }^{41}$

Sleep disturbance of sleep deprivation in bipolar disorder patients can trigger manic episodes in bipolar disorder patients. ${ }^{42}$ Partial sleep deprivation can also contribute to high rates of relapse for manic episodes. Although few studies have investigat- ed CBTI for bipolar disorder patients, it is recommended to avoid or use sleep restriction with caution. ${ }^{17}$

\section{Post-Traumatic Stress Disorder (PTSD)}

Post-traumatic stress disorder (PTSD) patients frequently experience sleep maintenance difficulties and nightmares. Insomnia symptoms and sleep disorders in PTSD patients maintain PTSD symptoms and are associated with significant stress and decreased mental and physical functioning. There have currently been three studies that have used CBTI in PTSD patients. ${ }^{43-45}$

All three studies used imagery rehearsal therapy (IRT) with PTSD patients. IRT is a treatment modality that helps patients remember recurrent nightmares and change the contents of the dream to be more benign. Afterwards, the patient is instructed to rehearse the "new" dream frequently during the day. All studies investigating IRT have found increased sleep quality and improved insomnia symptoms. Additionally, frequency of nightmares, PTSD symptoms, and symptoms of depression and anxiety have also decreased by treating the sleep problems (effect size 0.43-0.71).

\section{Alcohol Dependence}

Insomnia is a common symptom in patients with alcohol dependence when they are abstaining from alcohol ${ }^{46}$ Approximately $36-91 \%$ of alcohol dependence disorder patients report sleep disturbance for several weeks immediately after abstaining from alcohol, and a few studies report that sleep does not improve in some patients up to several months to several years. Currie et al. ${ }^{47}$ treated 60 patients with alcohol dependence using CBTI, and reported that their sleep quality and sleep efficiency improved, while wake after sleep onset and number of awakenings during the night decreased. However, it appears that insomnia treatment does not significantly affect the recurrence of alcohol dependence.

\section{THE EFFECTIVENESS OF USING CBTI FOR COMORBID PHYSICAL ILLNESSES}

\section{Chronic Pain}

Chronic pain is caused by diseases such as arthritis, fibromyalgia, herniated disc, or spinal stenosis. Approximately $50-88 \%$ of patients suffering from chronic pain experience sleep disorders. ${ }^{48,49}$ Research indicates that severity of sleep disturbance depends on pain severity. According to longitudinal studies investigating the bidirectional relationship between sleep and pain, pain interferes with sleep, and poor sleep subsequently increases the degree of pain experienced the following day.

Currently, four studies have investigated CBTI with chronic pain patients. Morin et al..$^{50}$ found that chronic pain patients 
who received CBTI took significantly less time to fall asleep, and treatment effects were maintained at 2 month and 6 month follow-up. Additionally, chronic pain patients reported improved mood, decreased anxiety, and decreased pain following CBTI. In another study conducted by Currie et al., ${ }^{47} 60$ chronic pain patients reported a significant improvement in sleep compared to the control group. Among the patients who received CBTI, $56 \%$ of patients reported having sleep efficiency (total sleep time / time spent in bed) of $85 \%$ or higher, and lower pain intensity compared to the control group. Decreased pain levels were maintained three months after receiving treatment, and the effect size for the treatment group was 0.56 , compared to 0.15 for the control group.

Edinger and colleagues conducted CBTI to fibromyalgia patients, and reported increased sleep quality and improved mood. ${ }^{51}$ However, CBTI did not decrease pain levels in these patients. Finally, Jungquist and colleagues compared chronic pain patients who received CBTI to a control group. ${ }^{52}$ The control group showed decreased sleep onset latency, wake after sleep onset, number of awakenings, and pain levels, and increased sleep efficiency compared to the control group.

\section{Cancer}

Approximately $30-50 \%$ of cancer patients report having insomnia, with breast cancer and lung cancer patients showing the highest prevalence. ${ }^{53}$ A majority of cancer patients experience insomnia symptoms when they are diagnosed with cancer, which is commonly due to psychological stress of being diagnosed with cancer. However, a significant number of these patients report experiencing insomnia symptoms years after being diagnosed, with insomnia symptoms persisting after receiving chemotherapy and even after their cancer has gone into remission. Additionally, a majority of cancer patients complain of experiencing fatigue through chemotherapy, along with decreased quality of life and increased depression and anxiety symptoms. Insomnia symptoms in cancer patients not only affects the patient's quality of life, but it also affects the progression of cancer. Research indicates that sleep deprivation weakens immune system functioning, which can especially have a negative effect on the effects of chemotherapy.

There are currently three studies that have investigated CBTI in cancer patients. Davidson and colleagues conducted CBTI over six sessions for cancer patients, using stimulus control, relaxation techniques, sleep hygiene, and cognitive techniques. ${ }^{53}$ Cancer patients reported increased sleep quality, sleep efficiency, and total sleep time, with decreased sleep onset latency, wake after sleep onset, and number of awakenings. Additionally, cancer patients reported decreased fatigue and increased role functioning following CBTI. Simeit and colleagues conducted relaxation techniques (progressive muscle relaxation vs. autogenics) over four weeks with cancer patients and compared treatment effects to a control group. ${ }^{54}$ Overall, total sleep time, sleep effi- ciency, and sleep quality improved, while sleep onset latency, use of sleep medication, and daytime fatigue decreased. Additionally, patients who received treatment reported a significant increase in quality of life compared to the control group. Quesnel and colleagues conducted CBTI in ten cancer patients, and found a significant decrease in wake after sleep onset, and an increase in sleep efficiency. ${ }^{55}$ Depression and fatigue decreased and quality of life increased following CBTI.

\section{DISCUSSION}

Cognitive-behavioral therapy for insomnia can be an alternative and non-pharmacological option for insomnia. Additionally, it can significantly contribute to the treatment effects of comorbid psychiatric illnesses when used as a supplementary and adjunctive treatment to the original disorder. Based on research that insomnia treatment can improve depression symptoms in the absence of depression-specific treatment, in addition to the results that insomnia treatment can prevent relapse of depressive episodes, insomnia treatment should be strongly recommended to depression patients who have comorbid insomnia. Additionally, based on research findings that insomnia treatment can decrease suicidal ideation of insomnia patients, CBTI should also be recommended to insomnia patients with high levels of suicidal ideation.

For PTSD patients, CBTI decreases frequency of nightmares and sleep maintenance difficulties, and is also effective in improving PTSD symptoms. CBTI can also alleviate insomnia symptoms in patients with alcohol dependence, but is not effective in decreasing symptoms associated with alcohol dependence. There are few studies that investigate CBTI in bipolar disorder patients. However, based on current research, it is advised to use sleep restriction with caution in this patient population.

For patients with physical illnesses, relieving insomnia symptoms through implementing CBTI can improve quality of life. CBTI can especially be helpful for patients who are limited in taking psychiatric medications due to risk of drug interactions from taking multiple medications for their original physical illness.

For patients with chronic pain, research has been consistent in showing that CBTI alleviates insomnia symptoms of patients with chronic pain. Research outcomes have been variable in showing that CBTI is helpful in decreasing pain, but generally the results have been optimistic. In cancer patients, CBTI helps decrease fatigue due to chemotherapy or from cancer by alleviating insomnia symptoms, and also helps increase quality of life. 


\section{CONCLUSION}

Cognitive-behavioral therapy for insomnia as an adjunctive treatment can help treatment effects of comorbid psychiatric and physical illnesses. In places like Korea, where seeking treatment for psychiatric illnesses is stigmatized, CBTI can act as an alternative and accessible treatment that leads to treatment for other psychiatric illnesses. Additionally, Korea's high rate of suicide among all OECD countries has become a mushrooming problem, and CBTI can help prevent suicides by decreasing insomnia symptoms, which has been noted as one of the biggest risk factors. Research henceforth should try to further investigate the effects of CBTI when applied to other comorbid psychiatric and physical illnesses.

\section{Conflicts of Interest}

The author has no financial conflicts of interest.

\section{REFERENCES}

1. Ford DE, Kamerow DB. Epidemiologic study of sleep disturbances and psychiatric disorders. An opportunity for prevention? JAMA 1989;262: 1479-84.

2. Ohayon MM, Caulet M, Lemoine P. Comorbidity of mental and insomnia disorders in the general population. Compr Psychiatry 1998;39: 185-97.

3. Ohayon MM, Roth T. What are the contributing factors for insomnia in the general population? J Psychosom Res 2001;51:745-55.

4. Ancoli-Israel S, Roth T. Characteristics of insomnia in the United States: results of the 1991 National Sleep Foundation Survey. I. Sleep 1999;22 Suppl 2:S347-53.

5. Ishigooka J, Suzuki M, Isawa S, Muraoka H, Murasaki M, Okawa M. Epidemiological study on sleep habits and insomnia of new outpatients visiting general hospitals in Japan. Psychiatry Clin Neurosci 1999;53: 515-22.

6. Simon GE, VonKorff M. Prevalence, burden, and treatment of insomnia in primary care. Am J Psychiatry 1997;154:1417-23.

7. Lee SH, Lee HS, Yoo KJ, Kim SH. Study on frequency of Adult Sleep Disorders. J Korean Med Assoc 1988;31:657-64.

8. Kuppermann M, Lubeck DP, Mazonson PD, Patrick DL, Stewart AL, Buesching DP, et al. Sleep problems and their correlates in a working population. J Gen Intern Med 1995;10:25-32.

9. Zammit GK, Weiner J, Damato N, Sillup GP, McMillan CA. Quality of life in people with insomnia. Sleep 1999;22 Suppl 2:S379-85.

10. Ohayon MM, Caulet M, Guilleminault C. How a general population perceives its sleep and how this relates to the complaint of insomnia. Sleep 1997;20:715-23.

11. Aldrich MS. Automobile accidents in patients with sleep disorders. Sleep 1989;12:487-94.

12. Balter MB, Uhlenhuth EH. New epidemiologic findings about insomnia and its treatment. J Clin Psychiatry 1992;53 Suppl:34-9; discussion 40-2.

13. Johnson LC, Spinweber CL. Good and poor sleepers differ in Navy performance. Mil Med 1983;148:727-31.

14. American Psychiatric Association. 2010. American Psychiatric Association DSM-5 Development [online]. Available from: http://www.dsm5. org/ProposedRevisions/Pages/proposedrevision.aspx?rid=65.

15. Wilson SJ, Nutt DJ, Alford C, Argyropoulos SV, Baldwin DS, Bateson AN, et al. British Association for Psychopharmacology consensus statement on evidence-based treatment of insomnia, parasomnias and circadian rhythm disorders. J Psychopharmacol 2010;24:1577-601.
16. Morin CM, Espie CA. Insomnia: a clinician's guide to assessment and treatment. New York: Springer Science \& Business Media 2003.

17. Smith MT, Huang MI, Manber R. Cognitive behavior therapy for chronic insomnia occurring within the context of medical and psychiatric disorders. Clin Psychol Rev 2005;25:559-92.

18. Smith MT, Perlis ML, Park A, Smith MS, Pennington J, Giles DE, et al. Comparative meta-analysis of pharmacotherapy and behavior therapy for persistent insomnia. Am J Psychiatry 2002;159:5-11.

19. Sivertsen B, Omvik S, Pallesen S, Bjorvatn B, Havik OE, Kvale G, et al. Cognitive behavioral therapy vs zopiclone for treatment of chronic primary insomnia in older adults: a randomized controlled trial. JAMA 2006;295:2851-8.

20. Morin CM, Bootzin RR, Buysse DJ, Edinger JD, Espie CA, Lichstein KL. Psychological and behavioral treatment of insomnia: update of the recent evidence (1998-2004). Sleep 2006;29:1398-414.

21. Morin CM, Gaulier B, Barry T, Kowatch RA. Patients' acceptance of psychological and pharmacological therapies for insomnia. Sleep 1992; 15:302-5.

22. Dolan DC, Taylor DJ, Bramoweth AD, Rosenthal LD. Cognitive-behavioral therapy of insomnia: a clinical case series study of patients with co-morbid disorders and using hypnotic medications. Behav Res Ther 2010;48:321-7.

23. Center NMHI. Suicide warning signs [online]. Substance Abuse and Mental Health Services Administration (SAMHSA). 2005. Available from: http://www.mentalhealth. samhsa.gov/publications/allpubs/walletcard/engwallet-card.asp.

24. Katz DA, McHorney CA. Clinical correlates of insomnia in patients with chronic illness. Arch Intern Med 1998;158:1099-107.

25. Smith MT, Perlis ML, Smith MS, Giles DE, Carmody TP. Sleep quality and presleep arousal in chronic pain. J Behav Med 2000;23:1-13.

26. Sridhar GR, Madhu K. Prevalence of sleep disturbances in diabetes mellitus. Diabetes Res Clin Pract 1994;23:183-6.

27. Nierenberg AA, Keefe BR, Leslie VC, Alpert JE, Pava JA, Worthington $\mathrm{JJ} 3$ rd, et al. Residual symptoms in depressed patients who respond acutely to fluoxetine. J Clin Psychiatry 1999;60:221-5.

28. Reynolds CF 3rd, Kupfer DJ. Sleep research in affective illness: state of the art circa 1987. Sleep 1987;10:199-215.

29. Spielman A, Anderson M. The clinical interview and treatment planning as a guide to understanding the nature of insomnia: the CCNY Insomnia Interview. In: Chorkroverty S. Sleep Disorders Medicine: Basic Science, Technical Considerations, and Clinical Aspects. 2nd ed. Woburn, MA: Butterworth-Heinemann 1999;385-426.

30. Ağargün MY, Kara H, Solmaz M. Sleep disturbances and suicidal behavior in patients with major depression. J Clin Psychiatry 1997;58:249-51.

31. Reynolds CF 3rd, Frank E, Houck PR, Mazumdar S, Dew MA, Cornes $\mathrm{C}$, et al. Which elderly patients with remitted depression remain well with continued interpersonal psychotherapy after discontinuation of antidepressant medication? Am J Psychiatry 1997;154:958-62.

32. Van Londen L, Molenaar RP, Goekoop JG, Zwinderman AH, Rooijmans HG. Three- to 5-year prospective follow-up of outcome in major depression. Psychol Med 1998;28:731-5.

33. Manber R, Edinger JD, Gress JL, San Pedro-Salcedo MG, Kuo TF, Kalista T. Cognitive behavioral therapy for insomnia enhances depression outcome in patients with comorbid major depressive disorder and insomnia. Sleep 2008;31:489-95.

34. Kuo T, Manber R, loewy D. Insomniacs with comorbid conditions achieved comparable improvement in a cognitive behavioral group treatment program as insomniacs without comorbid depression. Sleep 2001;14:A62.

35. Manber R, Loewy D, Kuo T, Black J, Palombini L, Koester U. The efficacy of cognitive behavioral group intervention in a tertiary setting. Sleep 2001;24:A63.

36. Fawcett J, Scheftner WA, Fogg L, Clark DC, Young MA, Hedeker D, et al. Time-related predictors of suicide in major affective disorder. Am J Psychiatry 1990;147:1189-94. 
37. Krakow B, Artar A, Warner TD, Melendrez D, Johnston L, Hollifield M, et al. Sleep disorder, depression, and suicidality in female sexual assault survivors. Crisis 2000;21:163-70.

38. Manber R, Bernert RA, Suh S, Nowakowski S, Siebern AT, Ong JC. CBT for insomnia in patients with high and low depressive symptom severity: adherence and clinical outcomes. J Clin Sleep Med 2011;7:645-52.

39. Association ASD. The International Classification of Sleep Disorders: Diagnostic and Coding Manual-revised. Rochester, MN: American Sleep Disorder Association 1997.

40. Jernajczyk W. Latency of eye movement and other REM sleep parameters in bipolar depression. Biol Psychiatry 1986;21:465-72.

41. Goldsmith DR, Wagstaff AJ, Ibbotson T, Perry CM. Spotlight on lamotrigine in bipolar disorder. CNS Drugs 2004;18:63-7.

42. Sachs GS. Unmet clinical needs in bipolar disorder. J Clin Psychopharmacol 2003;23(3 Suppl 1):S2-8.

43. Brower KJ, Aldrich MS, Robinson EA, Zucker RA, Greden JF. Insomnia, self-medication, and relapse to alcoholism. Am J Psychiatry 2001; 158:399-404.

44. Germain A, Shear MK, Hall M, Buysse DJ. Effects of a brief behavioral treatment for PTSD-related sleep disturbances: a pilot study. Behav Res Ther 2007;45:627-32.

45. Swanson LM, Favorite TK, Horin E, Arnedt JT. A combined group treatment for nightmares and insomnia in combat veterans: a pilot study. J Trauma Stress 2009;22:639-42.

46. Drummond SP, Gillin JC, Smith TL, DeModena A. The sleep of abstinent pure primary alcoholic patients: natural course and relationship to relapse. Alcohol Clin Exp Res 1998;22:1796-802.

47. Currie SR, Wilson KG, Pontefract AJ, deLaplante L. Cognitive-behavioral treatment of insomnia secondary to chronic pain. J Consult Clin Psychol 2000;68:407-16.

48. Morin CM, Gibson D, Wade J. Self-reported sleep and mood disturbance in chronic pain patients. Clin J Pain 1998;14:311-4.

49. Wilson KG, Watson ST, Currie SR. Daily diary and ambulatory activity monitoring of sleep in patients with insomnia associated with chronic musculoskeletal pain. Pain 1998;75:75-84.

50. Morin CM, Kowatch RA, Wade JB. Behavioral management of sleep disturbances secondary to chronic pain. J Behav Ther Exp Psychiatry 1989;20:295-302.

51. Edinger JD, Wohlgemuth WK, Krystal AD, Rice JR. Behavioral insomnia therapy for fibromyalgia patients: a randomized clinical trial. Arch Intern Med 2005;165:2527-35.

52. Jungquist CR, O’Brien C, Matteson-Rusby S, Smith MT, Pigeon WR, Xia Y, et al. The efficacy of cognitive-behavioral therapy for insomnia in patients with chronic pain. Sleep Med 2010;11:302-9.

53. Davidson JR, Waisberg JL, Brundage MD, MacLean AW. Nonpharmacologic group treatment of insomnia: a preliminary study with cancer survivors. Psychooncology 2001;10:389-97.

54. Simeit R, Deck R, Conta-Marx B. Sleep management training for cancer patients with insomnia. Support Care Cancer 2004;12:176-83.

55. Quesnel C, Savard J, Simard S, Ivers H, Morin CM. Efficacy of cognitive-behavioral therapy for insomnia in women treated for nonmetastatic breast cancer. J Consult Clin Psychol 2003;71:189-200. 